\title{
Nanomedicine for cutaneous tumors - lessons since the successful treatment of the Kaposi sarcoma
}

\author{
João Paulo F Longo*,1, Carolina M Lucci², Luís A Muehlmann³ \& Ricardo B Azevedo ${ }^{1}$ \\ ${ }^{1}$ Genetics \& Morphology Department, Institute of Biological Science, University of Brasilia, Brasília DF 70910-900, Brazil \\ ${ }^{2}$ Physiological Sciences Department, Institute of Biological Science, University of Brasilia, Brasília DF 70910-900, Brazil \\ ${ }^{3}$ Faculty of Ceilândia, University of Brasilia, Brasilia 72220-900, Brazil \\ *Author for correspondence: Tel.: +55 613107 3087; Fax: +55 6198143 2755; jplongo82@gmail.com
}
"Nowadays, the nanomedical literature has almost reached a consensus that translating data from preclinical animal models to the clinical and commercial use of nanomedicines is very difficult."

First draft submitted: 5 October 2018; Accepted for publication: 24 October 2018; Published online: 30 November 2018

Keywords: cancer $\bullet$ clinical trials $\bullet$ controlled drug release $\bullet$ dermatology $\bullet$ Doxil $\bullet$ EPR effect $\bullet$ Kaposi sarcoma $\bullet$ liposomes • nanomedicine $\bullet$ nanoparticles • oncology

Nanomedicine is an area of nanotechnology dedicated to the development of therapeutic and diagnostic solutions for medical challenges. This approach has been especially developed for oncology since the 1980s, and a huge number of papers have been published in the field since then. One of the highlights of this process was the release into the market of Doxil ${ }^{\circledR}$ (Titusville, NJ, USA) in the mid 1990s. However, after these 20-30 years of investigation, the scientific community and society in general expected that more nanomedical products would be released by the pharmaceutical industry and translated from 'bench to bedside'.

Nowadays, the nanomedical literature has almost reached a consensus that translating data from preclinical animal models to the clinical and commercial use of nanomedicines is very difficult. This translational problem mainly involves the histopathological organization of tumors developed by transplanted tumor cells used in preclinical studies, which is different from that of naturally occurring tumors. Some authors suggest that a 30 -year project should be planned to develop more realistic preclinical tumor models to test and improve the translation of innovative nanotechnologies for the general public [1,2].

A misconception that seems to have been in the minds of nanomedical investigators over recent decades was that all types of preclinical tumor models could behave in the same way as naturally occurring tumors. This erroneous concept increased the expectations of nanomedicine, and now the scientific community is facing a climate of mistrust related to nanomedicine [3].

The fact that preclinical tumors are not natural tumors is now a fairly obvious point. However, due to the absence of realistic models, researchers are still dependent on inoculated preclinical tumor models. As an example of this complexity, our research group has just published a report showing that a breast tumor line passively absorbs nanocarriers in a significantly different fashion when this cell line is inoculated, and is growing, in the subcutaneous tissue or when these cells migrate to the lungs, producing metastatic lesions [4]. In other words, the same tumor type can behave differently, depending on the stromal tissues surrounding it, subcutaneously or in the lung.

Thus, the development of new nanomedicines should consider the natural behavior of tumors, as well as the other interstitial and physiological interactions that these tumors have, to achieve the real positive effects of this innovative therapy in clinical practice. Therefore, the move from 'bench to bedside' requires a focus on specific tumor types, to optimize the translation process.

It is interesting to note that one of the first clinical trials using Doxil (1992-1994) succeeded in treating Kaposi's sarcoma, a cutaneous tumor that is very common in immunologically depressed HIV-positive patients. As the late 1980s was a period in which the spread of AIDS caused grave concern, Doxil emerged as an important 
nanomedicine option to treat this kind of cutaneous tumor [5,6]. The conclusion of the clinical trials was that Doxil was more effective and less toxic than conventional chemotherapy in the treatment of Kaposi's sarcoma.

All these positive results observed in the treatment of Kaposi's sarcoma reinforced the expectation that nanomedicines could act as 'magic bullets' to fight cancer, referring to Paul Ehrlich's well-known phrase [7]. In addition to these first clinical outcomes, thousands of preclinical studies demonstrated that the nanoparticles were passively accumulated by tumors, due to the classical enhanced permeability and retention (EPR) effect, further reinforcing this expectation $[1,4,8,9]$. Nowadays, Doxil is commercially used and presents superior performance over conventional therapies for the following tumor types: HIV-related Kaposi's sarcoma; metastatic breast cancer; recurrent ovarian cancer; and multiple myeloma [5].

A common point between Kaposi's sarcoma and most of the preclinical subcutaneous transplantable tumor models is the atypical proliferation of abnormal vascular structures with increased permeability [6,10], which makes them particularly favorable to the passive accumulation of nanocarriers, explained by the EPR effect. As a sarcoma, Kaposi's tumor has a mesenchymal origin and is highly vascularized, presenting aberrant blood vessels. On the other hand, cutaneous carcinomas, tumors with an epithelial origin, are poorly vascularized in comparison to Kaposi's sarcoma, and the vessels are not so permeable, which explains why there is poor passive accumulation of nanomedicines in cutaneous carcinomas in clinical conditions.

In conclusion, we believe that Kaposi's sarcoma serves as an example to explain that even tumors with the same anatomical location, such as cutaneous tumors, may exhibit distinct biological behavior depending on their embryonic origin. This distinct biological behavior significantly interferes with the passive delivery of nanocarriers to tumor tissues. Thus, in a prospective view toward future nanomedical developments, researchers should consider the biological behavior of each tumor type individually, and they should invest in alternative additive strategies in addition to the EPR effect. It is important to highlight that our research group truly believes that prospective approaches, such as the use of external active triggers [9], co-delivery of chemotherapeutical drugs [8], active delivery systems [11] and controlled-release systems, can offer possible effective solutions to improve cancer therapy using nanotechnology and nanocarriers.

\section{Financial \& competing interests disclosure}

The authors are grateful for financial support from the Brazilian agencies Conselho Nacional de Desenvolvimento Científico e Tecnológico (CNPq), Fundação de Amparo à Pesquisa do Distrito Federal (FAP/DF), Coordenação de Aperfeiçoamento de Pessoal de Nível Superior (CAPES) and the Alexander von Humboldt Foundation Program CONNECT. The authors have no other relevant affiliations or financial involvement with any organization or entity with a financial interest in or financial conflict with the subject matter or materials discussed in the manuscript apart from those disclosed.

No writing assistance was utilized in the production of this manuscript.

\section{References}

1. Wilhelm S, Tavares AJ, Dai Q et al. Analysis of nanoparticle delivery to tumours. Nat. Rev. Mater. doi:10.1038/natrevmats.2016.14 (2016) (Epub ahead of print).

2. Björnmalm M, Thurecht KJ, Michael M, Scott AM, Caruso F. Bridging bio-nano science and cancer nanomedicine. ACS Nano. 11(10), 9594-9613 (2017).

3. Moghimi SM, Farhangrazi ZS. Just so stories: the random acts of anti-cancer nanomedicine performance. Nanomedicine 10(8), 1661-1666 (2014).

4. Radicchi MA, Vaz de Oliveira J, Mendes ACP et al. Lipid nanoemulsion passive tumor accumulation dependence on tumor stage and anatomical location: a new mathematical model for in vivo imaging biodistribution study. J. Mater. Chem. B (2018) (In Press).

5. Barenholz YC. Doxil ${ }^{\circledR}$ - the first FDA-approved nano-drug: lessons learned. J. Control. Rel. 160(2), 117-134 (2012).

6. James N, Coker RJ, Tomlinson D et al. Liposomal doxorubicin (Doxil): an effective new treatment for Kaposi's sarcoma in AIDS. Clin. Oncol. 6(5), 294-296 (1994).

7. Strebhardt K, Ullrich A. Paul Ehrlich's magic bullet concept: 100 years of progress. Nat. Rev. Cancer 8(6), 473 (2008).

8. Ganassin R, Horst FH, Camargo NS et al. Selol nanocapsules with a poly(methyl vinyl ether-co-maleic anhydride) shell conjugated to doxorubicin for combinatorial chemotherapy against murine breast adenocarcinoma in vivo. Artif. Cells Nanomed. Biotechnol.(2018) (In Press).

9. Camara ALD, Nagel G, Tschiche HR et al. Acid-sensitive lipidated doxorubicin prodrug entrapped in nanoemulsion impairs lung tumor metastasis in a breast cancer model. Nanomedicine 12(15), 1751-1765 (2017). 
10. Longo JPF, de Melo LND, Mijan MC et al. Photodynamic therapy mediated by liposomal chloroaluminum-phthalocyanine induces necrosis in oral cancer cells. J. Biomater. Tissue Eng. 3(1), 148-156 (2013).

11. Ganassin R, Souza LR, Py-Daniel KR et al. Decoration of a poly (methyl vinyl ether-co-maleic anhydride)-shelled selol nanocapsule with folic acid increases its activity against different cancer cell lines in vitro. J. Nanosci. Nanotechnol. 18(1), 522-528 (2018). 
